\title{
Syphilis imitating urticarial vasculitis
}

\author{
Hideaki Miyachi MD PhD, Toshibumi Taniguchi MD PhD, Hiroyuki Matsue MD PhD
}

Cite as: CMAJ 2019 December 16;191:E1384. doi: 10.1503/cmaj.190469

See related article at www.cmaj.ca/lookup/doi/10.1503/cmaj.191075

A 27-year-old previously healthy woman presented with a 2-month history of recurrent raised pruritic erythematous plaques resembling urticaria that persisted for more than 24 hours (Figure 1). She was otherwise asymptomatic, with no history of fever or mucosal lesions. Previous treatments with orally administered $\mathrm{H}_{1}$ antihistamines and corticosteroids were ineffective. Skin biopsy showed perivascular inflammatory cell infiltration with some nuclear debris and swelling of endothelial cells involving postcapillary venules, whereas plasma cell infiltration was nearly absent (Appendix 1, Supplementary Figure 1, available at www. cmaj.ca/lookup/suppl/doi:10.1503/cmaj.190469/-/DC1). Direct immunofluorescence showed the deposition of complement and fibrinogen around blood vessels and in the dermal-epidermal junction, consistent with urticarial vasculitis. However, complement levels were unremarkable, and the $\mathrm{C} 1 \mathrm{q}$ solid-phase test for immune complexes was negative, excluding hypocomplementemic urticarial vasculitis. Autoantibodies associated with connective tissue diseases were not detected.

Serologic tests for HIV, hepatitis B virus and hepatitis $C$ virus were negative; however, the rapid plasma reagin test was positive (titer, 1:128), and the Treponema pallidum hemagglutination assay was reactive. Furthermore, immunohistochemistry with antiTreponema antibody showed marked invasion of Treponema into the epidermis, indicating that the skin lesions were caused by syphilis and confirming the diagnosis of secondary syphilis (Appendix 1, Supplementary Figure 2). With orally administered amoxicillin $(6 \mathrm{~g} / \mathrm{d})$ and probenecid $(1 \mathrm{~g} / \mathrm{d})$ for 4 weeks, the skin lesions resolved. This effective combination of drugs is used in Japan, as intramuscular benzathine penicillin is not available. ${ }^{1}$

Syphilis is still prevalent, with increasing incidence worldwide. ${ }^{2}$ Typical dermatologic findings of secondary syphilis are nonpruritic diffuse macules or papules with palmoplantar involvement; however, the rash can be quite variable. ${ }^{2}$ Syphilis imitates various skin diseases, such as drug eruptions, psoriasis, pityriasis rosea and many others, making diagnosis challenging. ${ }^{3}$ This case highlights the importance of considering serologic testing for syphilis when patients present with skin lesions that may be atypical for syphilis. Furthermore, skin biopsy and immunohistochemistry with anti-Treponema antibody are useful for diagnosing cutaneous lesions of secondary syphilis, ${ }^{4}$ which may otherwise be misdiagnosed as a nonspecific rash.

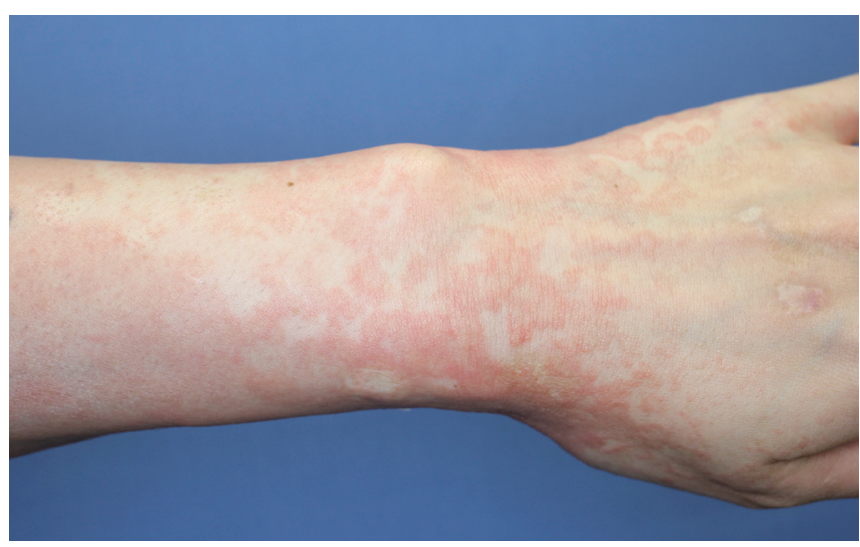

Figure 1: Raised pruritic erythematous plaques resembling urticaria in a 27-year-old woman with secondary syphilis.

\section{References}

1. Tanizaki R, Nichijima T, Aoki T, et al. High-dose oral amoxicillin plus probenecid is highly effective for syphillis in patients with HIV infection. Clin Infect Dis 2015; 61:177-83.

2. Hook EW 3rd. Syphilis [published erratum in Lancet 2019;393:986]. Lancet 2017;389:1550-7.

3. Cossman JP, Fournier JB. Frequency of syphilis diagnoses by dermatologists. JAMA Dermatol 2017;153:718-9.

4. Martín -Ezquerra G, Fernandez-Casado A, Barco D, et al. Treponema pallidum distribution patterns in mucocutaneous lesions of primary and secondary syphilis: an immunohistochemical and ultrastructural study. Hum Pathol 2009; 40:624-30.

Competing interests: Toshibumi Taniguchi accepts consultancy fees from ViiV Healthcare K.K., MSD K.K., Janssen Pharmaceutical K.K. and Torii Pharmaceutical Co. Ltd.; a research grant from MSD K.K.; and a speaking fee from MSD K.K., Torii Pharmaceutical Co. Ltd., Astellas Pharma Inc., Pfizer Japan Inc. and Shionogi \& Co., Ltd. No other competing interests were declared.

This article has been peer reviewed.

The authors have obtained patient consent.

Affiliations: Department of Dermatology (Miyachi, Matsue), Graduate School of Medicine; Department of Infectious Diseases (Taniguchi), Chiba University Hospital, Chiba-shi, Chiba, Japan

Correspondence to: Hideaki Miyachi, h-miyachi@umin.ac.jp 